\title{
Symplectic folding and nonisotopic polydisks
}

\author{
RICHARD HIND
}

Let $P_{1}$ be a polydisk and $P_{2}=\phi\left(P_{1}\right)$ where $\phi$ is a certain symplectic fold. We determine sharp lower bounds on the size of a ball containing the support of a symplectomorphism mapping $P_{1}$ to $P_{2}$. Optimal symplectomorphisms are the folds themselves. As a result, we construct symplectically nonisotopic polydisks in balls and in the complex projective plane.

53D35, 57R17; 53D42

\section{Introduction}

\subsection{Main results}

Our main result demonstrates the existence of different Hamiltonian isotopy classes of symplectically embedded polydisks inside a 4 -ball, and by the same argument also in the complex projective plane. Furthermore, we find exactly how large the ball can be before the embeddings become isotopic; the optimal isotopy is a version of symplectic folding.

Before stating the result precisely we fix some notation. We work in $\mathbb{R}^{4}$ with coordinates $\left(x_{1}, y_{1}, x_{2}, y_{2}\right)$ and standard symplectic form $\omega=\sum_{i} d x_{i} \wedge d y_{i}$. We denote by $B(a)$ the open ball of capacity $a$, that is

$$
B(a)=\left\{\pi \sum_{i}\left(x_{i}^{2}+y_{i}^{2}\right)<a\right\} \subset \mathbb{R}^{4}
$$

with the restricted symplectic form. The polydisk $P(a, b)$ is defined by

$$
P(a, b)=\left\{\pi\left(x_{1}^{2}+y_{1}^{2}\right) \leq a, \pi\left(x_{2}^{2}+y_{2}^{2}\right) \leq b\right\} \subset \mathbb{R}^{4} .
$$

For the results in this section, when describing a polydisk we will always assume that $a<b$. Sometimes $D(a)$ will be used to denote the closed disk of area $a$ in $\mathbb{R}^{2}$. The inclusion map then gives a symplectic embedding $P(a, b) \rightarrow B(R)$ for any $R>a+b$.

Let $H_{t}: \mathbb{R}^{4} \rightarrow \mathbb{R}, 0 \leq t \leq 1$ be a smooth family functions such that the map $\mathbb{R}^{4} \times[0,1] \rightarrow \mathbb{R},(x, t) \mapsto H_{t}(x)$ has compact support. The forms $d H_{t}$ are dual to 
vector fields $X_{H_{t}}$ under the symplectic form, that is, we define $X_{H_{t}}$ by $\left.X_{H_{t}}\right\rfloor \omega=d H_{t}$. Then the Hamiltonian diffeomorphism generated by $\left\{H_{t}\right\}$ is the time-1 flow (which exists by our assumption of compact support) of the time-dependent vector field $X_{H_{t}}$. Hamiltonian diffeomorphisms are symplectic. On the other hand, by a theorem of Gromov [7] a compactly supported symplectomorphism of $B(a)$ is Hamiltonian.

Here is the first part of our main result.

Theorem 1.1 Let $S<a+b<R \leq 2 a+b$. Then there does not exist a Hamiltonian diffeomorphism $\phi$ with support contained in $B(R)$ such that $\phi(P(a, b)) \subset B(S)$.

In cases when the ratio $b / a$ is sufficiently large there do exist Hamiltonian diffeomorphisms $\psi$ of $\mathbb{R}^{4}$ such that $\psi(P(a, b)) \subset B(S)$ for $S<a+b$. In fact, as the ratio $b / a \rightarrow \infty$ we may take $S \rightarrow \sqrt{a b}$, that is, the image of the polydisk can occupy an arbitrarily large proportion of the volume of the ball. For this, see for example Schlenk [18].

Hence, together with Gromov's theorem mentioned above we can produce examples of the following.

Corollary 1.2 Suppose that $a+b<R \leq 2 a+b$, so in particular $P(a, b) \subset B(R)$, and $b / a$ is very large. Then there exist symplectic embeddings $P(a, b) \rightarrow B(R)$ which do not extend to symplectomorphisms $B(R) \rightarrow B(R)$.

On the other hand, the second Ekeland-Hofer capacity of $P(a, b)$ is $2 a$ whereas the second Ekeland-Hofer capacity of $B(S)$ is $S$ (see Ekeland and Hofer [4]) and these capacities are monotonic under symplectic embeddings. Hence if $a=b$ then there are no symplectic embeddings of $P(a, b)$ into a $B(S)$ with $S<2 a=a+b$.

The easiest way to construct an embedding $P(a, b) \rightarrow B(S)$ for $S<a+b$ (when such an embedding exists) is via symplectic folding, and we shall see that Theorem 1.1 is sharp in the sense that this method applies whenever $R>2 a+b$.

Theorem 1.3 Let $R>2 a+b$. For any $\epsilon>0$ there exists a Hamiltonian diffeomorphism $\phi$ with compact support in $B(R)$ such that $\phi(P(a, b)) \subset B(2 a+b / 2+\epsilon)$.

Let us consider symplectic embeddings $f: P(a, b) \rightarrow B(R)$ under the equivalence relation that $f_{1} \sim f_{2}$ if there exists a Hamiltonian diffeomorphism $\phi$ with support in $B(R)$ such that $\phi \circ f_{1}=f_{2}$.

Hamiltonian diffeomorphisms $\phi$ with support in $B(R)$ can be generated by functions which all have support in $B(R)$. This follows directly from Gromov's theorem on 
symplectomorphisms of the ball, but also follows from simply rescaling as we assume our time-dependent Hamiltonian functions have (uniformly) compact support. Indeed, write $\phi=\phi_{1}$ where $\phi_{t}$ for $0 \leq t \leq 1$ is the Hamiltonian flow generated by functions $H_{t}$ with support in a fixed compact set $K$. Without loss of generality we may assume that $H_{t} \equiv 0$ when $t \notin[\epsilon, 1-\epsilon]$, so $\phi_{t}=\mathrm{id}$, the identity map, when $t<\epsilon$ and $\phi_{t}=\phi$ when $t>1-\epsilon$. Let $s:[0,1] \rightarrow[1, \infty)$ be a smooth function with $s(t)=1$ for $t$ close to 0 and 1 , and $M_{s}$ denote multiplication by $s$ in $\mathbb{R}^{4}$. Then if $s(t)$ is sufficiently large on the interval $[\epsilon, 1-\epsilon]$ we will have $K \subset M_{s(t)}(B(R))$ and so the flow $\tilde{\phi}_{t}:=M_{1 / s(t)} \circ \phi_{t} \circ M_{s(t)}$ will be Hamiltonian with compact support in $B(R)$ and $\widetilde{\phi}_{1}=\phi$.

Given this, we call the equivalence classes Hamiltonian isotopy classes. Then combining Theorem 1.3 with Theorem 1.1 we can say the following, making Corollary 1.2 more precise.

Corollary 1.4 Suppose that $2 a<b$ and $a+b<R \leq 2 a+b$. Then there exists at least two Hamiltonian isotopy classes of polydisks $P(a, b)$ inside $B(R)$.

Two isotopy classes are that of the inclusion and the inclusion composed with the symplectic fold of Theorem 1.3. The condition $2 a<b$ implies that for $\epsilon$ sufficiently small $2 a+b / 2+\epsilon<a+b$ and so the image of the fold lies in $B(a+b) \subset B(R)$.

Organization In Section 2 we study holomorphic curves in manifolds with cylindrical ends in order to prove Theorem 1.1. The technique of symplectic folding is now fairly well known. It was introduced by Lalonde and McDuff in [14] and studied extensively in [18], but in Section 3 we review the construction and show that it implies Theorem 1.3.

\subsection{Related work}

1.2.1 Isotopies of polydisks There are known to be nonisotopic polydisks embedded in larger polydisks. Observe that if $a, b<1$ then the polydisks $P(a, b)$ and $P(b, a)$ both lie in $P(1,1)$. We consider these as giving two embeddings $g_{1}, g_{2}: P(a, b) \rightarrow P(1,1)$. For any $R>a+b$, there exists a unitary transformation mapping $P(a, b)$ to $P(b, a)$ generated by a Hamiltonian with support contained in $B(R) \subset P(R, R)$. The following theorem is due to A Floer, H Hofer and K Wysocki.

Theorem 1.5 (Floer-Hofer-Wysocki [6, Theorem 4]) When $a, b<1$ and $a+b>1$ there does not exist a Hamiltonian diffeomorphism $\phi$ with support contained in $P(1,1)$ such that $\phi \circ g_{1}=g_{2}$. 
In other words, if the ambient square polydisk does not contain the trace of a rotation moving $P(a, b)$ onto $P(b, a)$ then the polydisks are not isotopic under any Hamiltonian flow. We contrast this with our Theorems 1.1 and 1.3 in the case when $2 a<b$. Here the polydisks are the image $P_{1}$ of the inclusion of $P(a, b)$ and the result $P_{2}$ of a fold described by Theorem 1.3 applied to $P_{1}$. By Theorem 1.3 the fold can be chosen to have support contained in an arbitrarily small neighborhood of the closed ball $\overline{B(2 a+b)}$ and the image $P_{2}$ can be chosen to lie in a small neighborhood of $\overline{B(2 a+b / 2)}$. But as $2 a<b$ we have $\overline{B(2 a+b / 2)} \subset B(a+b)$ and so Theorem 1.1 says that there is no Hamiltonian diffeomorphism with support in the open ball $B(2 a+b)$ mapping $P_{1}$ to $P_{2}$.

Theorem 1.5 was established as an application of symplectic homology, although as pointed out by K Ono it can also be derived from Floer theoretic methods applied to the distinguished boundary $\partial D(a) \times \partial D(b) \subset \partial D(a, b)$ of the polydisk, which is a Lagrangian torus.

1.2.2 Isotopies of ellipsoids Besides polydisks, another convenient class of domains used to investigate symplectic embedding problems are ellipsoids. The ellipsoid $E(a, b)$ is defined by

$$
E(a, b)=\left\{\frac{\pi\left(x_{1}^{2}+y_{1}^{2}\right)}{a}+\frac{\pi\left(x_{2}^{2}+y_{2}^{2}\right)}{b} \leq 1\right\} \subset \mathbb{R}^{4} .
$$

In particular $E(a, a)=\bar{B}(a)$. The ellipsoid $E(a, b)$ is embedded in the ball $B(R)$ by inclusion whenever $R>\max \{a, b\}$. In contrast to Theorem 1.1 however, according to a theorem of D McDuff this is the only isotopy class inside the ball. Indeed, this remains the case if we study any embedding from an ellipsoid to an ellipsoid.

Theorem 1.6 (McDuff [15, Corollary 1.6]) The space of symplectic embeddings $E(a, b) \rightarrow E^{\circ}\left(a^{\prime}, b^{\prime}\right)$ is path connected whenever it is nonempty.

Acknowledgements The author would like to thank Felix Schlenk for reading the paper and for some useful suggestions.

\section{Restriction on isotopies}

In this section we establish our constraint on the Hamiltonian diffeomorphisms of the standard polydisk $P(a, b) \subset B(R)$ for some $a+b<R<2 a+b$. (The case of $R=2 a+b$ will then follow trivially as our balls are open.) Our techniques apply to smooth domains so first we approximate $P(a, b)$ by a slightly smaller domain $W$ 
with smooth boundary. We recall that a Hamiltonian diffeomorphism with support in $B(R)$ can be generated by a Hamiltonian flow also with support inside $B(R)$ (see the discussion in Section 1.1 following Theorem 1.3). As an isotopy of $P(a, b)$ restricts to one of $W$ constraints on isotopies of $W$ imply constraints for the polydisk.

\subsection{The smooth domain $W$ and orbits on the boundary}

Fix a small irrational $\epsilon>0$ and a $0<\delta<<\epsilon$ and let $x_{0}=(a-\epsilon b) /\left(1-\epsilon^{2}\right)$. Then let $f:[0, a] \rightarrow[0, b]$ be a smooth convex function with $f(x)=b-\epsilon x$ for $x<x_{0}-\delta$ and $f(x)=\frac{1}{\epsilon}(a-x)$ for $x>x_{0}+\delta$. Finally set $K(x, y)=y-f(x)$.

It is convenient to use symplectic polar coordinates on $\mathbb{R}^{4}$, so we set $R_{i}=\pi\left(x_{i}^{2}+y_{i}^{2}\right)$ and $\tan \frac{y_{i}}{x_{i}}=\theta_{i}$. Then we define our approximation $W$ to $P(a, b)$ by

$$
W=\left\{K\left(R_{1}, R_{2}\right) \leq 0\right\} .
$$

This is a smooth domain with contact-type boundary and Reeb flow $R_{W}$ generated by the Hamiltonian vector field of $K$, namely

$$
R_{W}=2 \pi\left(K_{R_{1}} \frac{\partial}{\partial \theta_{1}}+K_{R_{2}} \frac{\partial}{\partial \theta_{2}}\right),
$$

where $K_{R_{i}}$ denotes the partial derivative with respect to $R_{i}$ as usual.

The Reeb flow preserves the Lagrangian tori $\left\{\left(R_{1}, R_{2}\right)=\right.$ const $\}$ in $\partial W$ and using the coordinates $\left(\theta_{1}, \theta_{2}\right)$ we can identify these tori with a fixed torus $T^{2}$ and the integer homology with $H_{1}\left(T^{2}, \mathbb{Z}\right)=\mathbb{Z}^{2}$. Then we get closed orbits in a homology class $(m, n) \in H_{1}\left(T^{2}\right)$ exactly when $K_{R_{1}} / K_{R_{2}}=m / n$. By the definition of $K$, in our case $K_{R_{2}} \equiv 1$ and $K_{R_{1}}$ is a function only of $R_{1}$ and is increasing from $\epsilon$ to $\frac{1}{\epsilon}$ as $R_{1}$ moves through $x_{0}$. Therefore, given a rational $m / n \in\left[\epsilon, \frac{1}{\epsilon}\right]$ we will have a 1 -parameter family of closed Reeb orbits in the corresponding homology class lying in the torus over $\left(R_{1}, R_{2}\right)$ where $K_{R_{1}}=m / n$. As $\epsilon$ is irrational these are the only closed Reeb orbits in $\partial W$ except for covers of $\left\{R_{1}=0\right\}$ and $\left\{R_{2}=0\right\}$.

Definition 2.1 Given $m, n \in \mathbb{Z}_{\geq 0}$ with $m / n \in\left(\epsilon, \frac{1}{\epsilon}\right)$ let $\gamma_{m, n}$ denote a Reeb orbit in the corresponding homology class $(m, n)$. Thus if $m, n$ are coprime, $\gamma_{r m, r n}$ denotes an $r$-fold cover of a primitive Reeb orbit $\gamma_{m, n}$.

Let $\gamma_{1}^{r}$ denote the $r$-fold cover of $\gamma_{1}=\left\{R_{2}=0\right\}$ and $\gamma_{2}^{r}$ denote the $r$-fold cover of $\gamma_{2}=\left\{R_{1}=0\right\}$.

Now, if we fix a symplectic trivialization of $\left.T \mathbb{R}^{4}\right|_{\gamma}$, the tangent bundle of $\mathbb{R}^{4}$ restricted to a closed orbit $\gamma$ of $R$ of period $T$, then the derivative of the Reeb flow (extended to 
act trivially normal to $\partial W$ ) gives a map $\psi:[0, T] \rightarrow \operatorname{Symp}(4, \mathbb{R})$, where $\operatorname{Symp}(4, \mathbb{R})$ is the group of $4 \times 4$ symplectic matrices. Associated to such a path is a Conley-Zehnder index $\mu(\gamma)$ defined in this case by Robbin and Salamon in [17].

Lemma 2.2 With respect to the standard basis of $\mathbb{R}^{4}$ we have $\mu\left(\gamma_{i}^{r}\right)=2 r+1$, provided $r<\frac{1}{\epsilon}$, and $\mu\left(\gamma_{m, n}\right)=2(m+n)+\frac{1}{2}$.

Proof The computation for the orbits in the coordinate planes is contained for example in Hofer Wysocki and Zehnder [9, Lemma 1.6], and we omit that. Similar computations for the other orbits can be found in Bourgeois [1] (see also Hutchings and Sullivan [13]) but we review this anyway.

First of all, consider a different symplectic trivialization consisting of the Reeb vector field $R$ itself, a normal vector $n$ to $\partial W$, a vector $v$ perpendicular to $R$ and tangent to the Lagrangian torus, and a vector $w$ in $\partial W$ symplectically orthogonal to $n$ and $v$. The flow preserves $R$ and $n$ (by definition) and so the index is determined by its action on the symplectic complement $\langle v, w\rangle$. Restricted to this subspace the matrix $\psi(t)$ is of the form

$$
\psi(t)=\left(\begin{array}{ll}
1 & t \\
0 & 1
\end{array}\right)
$$

that is, $v$ is preserved (as the flow is linear on the Lagrangian fibers) but the image of $w$ has a component in the direction of $v$ due to the change in direction of $R$ as we vary the fibers. Thus the orbits $\gamma_{m, n}$ are said to be of hyperbolic type and the index with respect to this trivialization is $\frac{1}{2}$.

We wish to work with a different trivialization and so the matrix $\psi(t)$ above must be composed with a symplectic change of basis matrix $\zeta(t)$ taking the basis described above to the standard basis of $\mathbb{R}^{4}$. If we identify $\mathbb{R}^{4}$ with $\mathbb{C}^{2}$ then this change of basis is actually complex and so up to scale we can write $\zeta(t)$ in complex coordinates as

$$
\zeta(t)=\left(\begin{array}{cc}
i e^{i m t} & i e^{i m t} \\
i e^{i n t} & -i e^{i n t}
\end{array}\right), \quad 0 \leq t \leq 2 \pi .
$$

The path $\zeta(t)$ has Maslov index $2(m+n)$ and so by the catenation formula for ConleyZehnder indices (see [17, Theorem 2.3]) with respect to our chosen trivialization we have $\mu\left(\gamma_{m, n}\right)=2(m+n)+\frac{1}{2}$.

\subsection{Holomorphic curves in $\mathbb{C P} P^{2} \backslash W$}

Let $\mathbb{C} P^{2}(R)$ denote the complex projective plane with its Fubini-Study symplectic form scaled such that lines have area $R$. There exists a natural symplectic embedding 
$B(R) \subset \mathbb{C} P^{2}(R)$ whose image is the complement of $\mathbb{C} P^{1}(\infty)$, the line at infinity. In order to study holomorphic curves, we can therefore think of $W$ as embedded $W \subset B(R) \subset \mathbb{C} P^{2}(R)$.

2.2.1 The almost-complex structure on $X$ and finite energy curves A neighborhood of $\partial W \subset \mathbb{C} P^{2}(R)$ is symplectomorphic to $\partial W \times(-\epsilon, \epsilon)$ with symplectic form $d\left(e^{s} \lambda\right)$, where $\lambda$ is a contact form on $\partial W$ and $s$ is the coordinate on $(-\epsilon, \epsilon)$. Indeed, let $Z$ denote the radial vector field

$$
Z=2 \sum R_{i} \frac{\partial}{\partial R_{i}}
$$

on $\mathbb{R}^{4}$. This is a Liouville vector field transverse to $\partial W$, that is, $\mathcal{L}_{Z} \omega=\omega$. Then $\sigma=Z\rfloor \omega$ is a primitive of $\omega$, and we also have $\mathcal{L}_{Z} \sigma=\sigma$. Note that $\sigma(Z)=0$. We identify a neighborhood of $\partial W=\partial W \times\{0\}$ with $\partial W \times(-\epsilon, \epsilon)$ such that $Z$ is given by $\partial / \partial s$, where $s$ is the coordinate on $(-\epsilon, \epsilon)$. Then in these coordinates we can write $\sigma=e^{s} \lambda$ where $\lambda$ is the restriction of $\left.\sigma=Z\right\rfloor \omega$ to $\partial W$. Let $\xi=\{\lambda=0\}$ be our corresponding contact structure on $\partial W$.

Now, following Eliashberg, Givental and Hofer [5] we will study holomorphic curves in

$$
X=\left(\mathbb{C} P^{2}(R) \backslash W\right) \cup(\partial W \times(-\infty, 0]),
$$

where the gluing is defined using the above identification of a neighborhood of $\partial W$ with $\partial W \times(-\epsilon, \epsilon)$. The symplectic form on $\mathbb{C} P^{2}(R) \backslash W$ extends smoothly to all of $X$ after a small perturbation near $\partial W$ by the formula $\omega=d(\phi(t) \lambda)$ where $\phi$ is an increasing function with $\phi(t)=e^{t}$ for $t$ close to $\epsilon$ and $\phi(t) \rightarrow 1$ as $t \rightarrow-\infty$. We associate a tame almost-complex structure $J$ which is translation invariant outside of a compact set and maps the Reeb vector $R_{W}$ to $-\partial / \partial s$. We also assume that $J$ preserves the contact planes $\xi$. The symplectic form is chosen so that $X$ is symplectomorphic to $\mathbb{C} P^{2}(R) \backslash W$, but the description of the complex structure on the cylindrical end is clearer in terms of $X$.

There is a theory of holomorphic curves mapping Riemann surfaces with punctures into $X$, which are asymptotic at their punctures to cylinders $\gamma \times(-\infty, 0)$, where $\gamma$ is a closed Reeb orbit in $\partial W$. These are sometimes called finite energy curves. The literature is fairly extensive, with some fundamental works including Hofer, Wysocki and Zehnder $[9 ; 11 ; 10 ; 12]$. Of course, under the symplectomorphism from $X$ to $\mathbb{C} P^{2}(R) \backslash W$ we could also think of holomorphic curves with image in $\mathbb{C} P^{2}(R) \backslash W$. In this case, the curves extend to maps from the oriented blow up of the Riemann surface at its punctures, mapping the boundary circles to closed Reeb orbits on $\partial W$; see Bourgeois, Eliashberg, Hofer, Wysocki and Zehnder [3, Proposition 5.10]. 
2.2.2 Area and index formulas for degree 1 curves The punctures of a finite energy holomorphic curve are called elliptic if they are asymptotic to a $\gamma_{i}^{r}$ or hyperbolic if they are asymptotic to a $\gamma_{m, n}$. We can define the Chern class $c_{1}(C)$ of such a curve $C$ to be $3 d$, where $d$ is the degree, that is, the degree of the 2 -cycle in $\mathbb{C} P^{2}$ formed by gluing disks in $W$ to the asymptotic limits of $C$ on $\partial W$. This is exactly the number of zeros of a section of the determinant line bundle $\left.\Lambda^{2}(T X, J)\right|_{C}$ which agrees with a trivial section of $\mathbb{C}^{2}$ near the punctures.

Suppose that a curve $C$ of degree $d$ has $e_{1}$ punctures asymptotic to orbits $\gamma_{1}^{r_{i}}$ for $1 \leq i \leq e_{1}$ and $e_{2}$ punctures asymptotic to orbits $\gamma_{2}^{s_{j}}$ for $1 \leq j \leq e_{2}$. Also, suppose the curve has $h$ hyperbolic punctures asymptotic to $\gamma_{m_{k}, n_{k}}$ for $1 \leq k \leq h$ respectively.

We recall from Section 2.1 that $\delta$ is a very small parameter chosen to control the approximation of our rounded domain $W$ to $P(a, b)$.

Lemma 2.3 The symplectic area of $C$ is given by

$$
\int_{C} \omega=d R-\sum_{i=1}^{e_{1}} r_{i} a-\sum_{j=1}^{e_{2}} s_{j} b-\sum_{k=1}^{h}\left(m_{k} a+n_{k} b\right)+\delta(C),
$$

where $\delta(C)$ is an error term of order $\delta$.

Proof A Reeb orbit $\gamma_{m, n} \subset \partial W$ lying over some $\left(R_{1}, R_{2}\right)$ bounds a disk in $W$ of area $m R_{1}+n R_{2}$. Due to our rounding of the polydisk all hyperbolic orbits lie over radial coordinates which are approximately $(a, b)$. The difference between the actual radial coordinates and $(a, b)$ accounts for the error term in our formula.

Given this, each of the negative terms in the formula correspond roughly to the areas of disks which can be glued to $C$ (thought of now as lying in $\mathbb{C} P^{2}(R) \backslash W$ ) to produce a closed cycle in $\mathbb{C} P^{2}$ of degree $d$ and hence area $d R$.

Remark 2.4 We observe immediately from Lemma 2.3 that (nontrivial) curves of nonpositive degree have negative area and so cannot exist for a tame almost-complex structure.

Under the assumption that $a+b<R<2 a+b$ we now document all genus 0 holomorphic curves of degree 1 satisfying a certain area restriction. The motivation for the restriction is that such curves can appear as boundary components of a certain moduli space of finite energy planes which will be the basis of our proof of Theorem 1.1. We will henceforth also use $\delta$ to denote the maximum of the possible error terms in Lemma 2.3 and assume it is chosen very small, in particular $\delta<2 a+b-R$. 
Lemma 2.5 Finite energy curves in $X$ of degree 1 and area at most $R-a-b+\delta$ are of one of the following types:
(I) $e_{2}=h=0,1+b / a \leq \sum_{1}^{e_{1}} r_{i}<2+b / a$;
(II) $e_{1}=h=0, e_{2}=1, s_{1}=2$;
(III) $e_{1}=h=0, e_{2}=2, s_{1}=s_{2}=1$;
(IV) $e_{1}=e_{2}=1, h=0, r_{1}=s_{1}=1$;
(V) $e_{1}=e_{2}=0, h=1, m_{1}=n_{1}=1$.

Proof The area formula of Lemma 2.3 implies that for such a curve we must have

$$
-\delta<R-\sum_{i=1}^{e_{1}} r_{i} a-\sum_{j=1}^{e_{2}} s_{j} b-\sum_{k=1}^{h}\left(m_{k} a+n_{k} b\right) \leq R-a-b+\delta,
$$

which, as $R<2 a+b$, implies

$$
a+b-\delta \leq \sum_{i=1}^{e_{1}} r_{i} a+\sum_{j=1}^{e_{2}} s_{j} b+\sum_{k=1}^{h}\left(m_{k} a+n_{k} b\right)<2 a+b
$$

when $\delta$ is sufficiently small. Now, recalling that the $r_{i}, s_{j}, m_{k}$ and $n_{k}$ are all nonnegative integers we can simply check that the only possibilities are those described, at least provided that $\delta$ is chosen sufficiently small.

Remark 2.6 As holomorphic curves have positive area, curves of types (II) and (III) are possible only if $b<2 a$.

Moduli spaces of finite energy curves, that is, spaces of such curves of a certain type modulo reparameterizations of the underlying Riemann surface, have a virtual index, and we now compute this for the types of curves appearing in Lemma 2.5.

Lemma 2.7 The virtual index of the curves described in Lemma 2.5 are as follows:

(I) $\quad$ index $=4-2 \sum r_{i} \leq-2$;

(II) index $=0$;

(III) index $=0$;

(IV) index $=0$;

(V) index $=1$. 
Proof The general index formula is

$$
\begin{aligned}
\operatorname{index}(C)=e_{1}+e_{2}+h-2+2 c_{1}(C)-\sum_{i=1}^{e_{1}} \mu\left(\gamma_{1}^{r_{i}}\right) & -\sum_{j=1}^{e_{2}} \mu\left(\gamma_{2}^{s_{j}}\right) \\
& -\sum_{k=1}^{h}\left(\mu\left(\gamma_{m_{k}, n_{k}}\right)-\frac{1}{2} \operatorname{dim} V_{k}\right) .
\end{aligned}
$$

For this, see Bourgeois [2]. The formula follows from Schwarz [19] together with the characterization of the Conley-Zehnder index in [17]. Here $c_{1}(C)$ is the Chern class which we have normalized to be 3 for degree 1 curves, and $\operatorname{dim} V_{k}$ is the dimension of the family of hyperbolic orbits containing $\gamma_{m_{k}, n_{k}}$, namely $\operatorname{dim} V_{k}=1$. Substituting the Conley-Zehnder indices from Lemma 2.2 we get

$$
\operatorname{index}(C)=4+e_{1}+e_{2}+h-\sum_{i=1}^{e_{1}}\left(2 r_{i}+1\right)-\sum_{j=1}^{e_{2}}\left(2 s_{j}+1\right)-\sum_{k=1}^{h} 2\left(m_{k}+n_{k}\right),
$$

or equivalently

$$
\operatorname{index}(C)=4+h-2 \sum_{i=1}^{e_{1}} r_{i}-2 \sum_{j=1}^{e_{2}} s_{j}-2 \sum_{k=1}^{h}\left(m_{k}+n_{k}\right) .
$$

The formulas follow readily from here. For the inequality on the index for curves of type (I) we note from Lemma 2.5 that $\sum r_{i} \geq 1+b / a$ and so the index satisfies

$$
\text { index }=4-2 \sum r_{i} \leq 4-2\left(1+\frac{b}{a}\right)=2\left(1-\frac{b}{a}\right) \leq-2,
$$

since we always assume $b>a$.

\subsection{Proof of Theorem 1.1}

Our method of proof is to construct a moduli space of holomorphic curves $\mathcal{M}_{t}$ for $0 \leq t \leq 1$ corresponding to each polydisk (or rather copy of $W$ ) in a symplectic isotopy; $\mathcal{M}_{0}$ will relate to the standard polydisk and we shall argue by contradiction and suppose that $\mathcal{M}_{1}$ corresponds to a symplectically isotopic polydisk in $B(S)$. We will show that $\mathcal{M}_{0}$ is nonempty (and in fact represents a nontrivial cobordism class) while $\mathcal{M}_{1}$ is empty. The key to the proof is then that $\bigcup_{t} \mathcal{M}_{t}$ gives a compact cobordism between $\mathcal{M}_{0}$ and $\mathcal{M}_{1}$ provided all polydisks lie in $B(R)$ or $\mathbb{C} P^{2}(R)$ for some $R<2 a+b$. This gives a contradiction. If $R>2 a+b$ then the universal moduli space is noncompact; see Remark 2.16 at the end of this section. 
2.3.1 Existence of finite energy curves Here we establish the following. Recall from Lemma 2.5 that curves of type (V) are finite energy planes (that is, curves with domain the complex plane $\mathbb{C}$ ) of degree 1 which are asymptotic at infinity to an orbit of type $\gamma_{1,1}$.

Lemma 2.8 There exists a compatible almost-complex structure $J_{0}$ on $X$ such that there exist $J_{0}$ holomorphic finite energy curves of type (V).

Proof We can produce finite energy curves in the manifold $X$ by starting with curves in $\mathbb{C} P^{2}$ and stretching the neck along $\partial W$. This means that we start with a tame almost-complex structure restricting to a translation invariant structure on $\partial W \times(-\epsilon, \epsilon) \subset \mathbb{C} P^{2}$ satisfying $J(R)=-\partial / \partial t$ and replace it by a sequence of tame almost-complex structures $J_{N}$ satisfying $J_{N}(R)=-\frac{1}{N} \partial / \partial t$, but such that $J_{N}=J$ on the contact planes $\xi$, which are preserved. For this see [3, Section 3.4]. We can choose embeddings $\phi_{N}$ of $\mathbb{C} P^{2}(R) \backslash W$ into $X$ pushing forward $J_{N}$ to an almost-complex structure which on increasingly large subsets of the cylindrical end $\partial W \times(-\infty, 0] \subset X$ is translation invariant and satisfies $J(R)=-\partial / \partial t$.

Now, there exists a unique pair $\left(R_{1}, R_{2}\right)$ with $K\left(R_{1}, R_{2}\right)=0$ and $K_{R_{1}}=K_{R_{2}}$; see the definitions at the start of Section 2.1. In the corresponding Lagrangian fiber the Reeb flow is generated by $\partial / \partial \theta_{1}+\partial / \partial \theta_{2}$. Let $c \in \mathbb{C}$ satisfy $|c|^{2}=R_{2} / R_{1}$. Then any complex line $z_{2}=c z_{1}$ (thought of as the affine part of a sphere in $\mathbb{C} P^{2}(R)$ ) is tangent to both the radial vector field $Z$ (that is, $\partial / \partial t$ in the local coordinates on the neck region) and to the Reeb vector field on $\partial W$. Thus, if we choose our initial almost-complex structure $J$ such that these curves are indeed holomorphic, then they will remain holomorphic during our stretching construction. Therefore, in the limit as $N \rightarrow \infty$ (see [3]) such curves converge to a holomorphic building whose component in $X$ is a degree 1 finite energy plane asymptotic to an orbit $\gamma_{1,1}$. Denote by $J_{0}$ the limiting almost-complex structure on $X$.

2.3.2 Moduli spaces Let us fix an orbit $\eta$ of type $\gamma_{1,1}$. Consider the moduli space

$$
\mathcal{M}_{0}=\left\{u: \mathbb{C} \rightarrow X \mid \operatorname{degree}(u)=1, \bar{\partial}_{J_{0}} u=0, u \sim \eta\right\} / G,
$$

where $u \sim \eta$ means that $u$ is asymptotic at infinity to $\eta$, and $G$ is the reparameterization group of $\mathbb{C}$.

Automatic regularity is a very useful tool for studying moduli spaces of holomorphic curves in 4-dimensional manifolds. In the closed case this is due to Gromov [7, 2.1.C1]; see also Hofer, Lizan and Sikorav [8]. In the situation of finite energy curves the result is due to C Wendl and can be stated as follows. Actually, Wendl's result is more general; 
it includes the case of curves with both punctures and boundary components, but in this paper we consider only curves with punctures.

Theorem 2.9 (Wendl [20, Theorem 1]) Let $u$ be a finite energy curve in an almostcomplex 4-manifold with cylindrical ends and suppose that

$$
\operatorname{index}(u)>c_{N}(u)+Z(d u) .
$$

Then $u$ is regular.

The various terms in Theorem 2.9 require some explanation. First we need to define $u$ as a class in a certain moduli space of finite energy curves. This moduli space can consist of curves with exactly the same asymptotic limits (as in the case of our $\mathcal{M}_{0}$ ) or we could allow the asymptotic limits to vary in a Morse-Bott family. Given this, index $(u)$ denotes the virtual dimension of a moduli space. In the case of $\mathcal{M}_{0}$ this virtual index is 0 . This follows from Lemma $2.7(\mathrm{~V})$. The index is 0 here rather than 1 as we are requiring our curves to be asymptotic to a specific orbit $\eta$, rather than any orbit of the type $\gamma_{1,1}$.

The term $Z(d u)$ is the sum of the critical points of $u$ counted with multiplicity. Here we note that curves in $\mathcal{M}_{0}$ are necessarily embedded and so the corresponding number $Z(d u)$ is 0 . To see this, observe that if we think of the image of $u$ as lying in $\mathbb{C} P^{2}(R) \backslash W$ then we can glue to this image a disk roughly defined by $\{(a z, b z)|| z \mid \leq 1\} \subset \mathbb{C}^{2}$ to produce a 2 -cycle $C$ in $\mathbb{C} P^{2}$. The exponential approach of $u$ to its asymptotic limit means that it is easy to smooth $C$ near the glued boundary to form a cycle which is a smooth symplectic submanifold near $W$ and coincides with the image of $u$ elsewhere. Thus $J_{0}$ can be perturbed near $\partial W$ and then extended to a tame almost-complex structure on $\mathbb{C} P^{2}(R)$ for which the cycle $C$ is holomorphic, indeed the image of a holomorphic sphere. As $C$ has degree 1 it is somewhere injective and so the adjunction formula (see McDuff and Salamon [16, Theorem 2.6.4]) implies that $C$ is the image of an embedding and hence $u$ has no critical points.

Finally, $c_{N}(u)$ is the half integer valued normal first Chern number defined by

$$
2 c_{N}(u)=\operatorname{index}(u)-2+2 g+\sharp \Gamma_{0} .
$$

This is [20, Formula (1.2)] in the case of finite energy curves without boundary components. Here $g$ is the genus of the curve, which in our case is 0 , and $\sharp \Gamma_{0}$ is the number of punctures of $u$ asymptotic to so-called even orbits. So in the case of finite energy planes, which have only one end, we have $2 c_{N}(u) \leq \operatorname{index}(u)-1$.

Putting everything together, in our situation we have index $(u)=0, Z(d u)=0$ and $c_{N}(u) \leq-1 / 2$. Thus the hypotheses of Theorem 2.9 are satisfied. The conclusion is 
that curves $u$ in $\mathcal{M}_{0}$ are regular. This means that the linearized Cauchy-Riemann operator at $u$ is surjective and hence that $\mathcal{M}_{0}$ has the structure of a manifold of dimension 0 near $u$.

We recall that $\mathcal{M}_{0}$ is nonempty by Lemma 2.8 and so can summarize our findings as follows.

Lemma 2.10 The moduli space $\mathcal{M}_{0}$ is a nonempty manifold of dimension 0 .

Arguing by contradiction, suppose that we have a symplectic isotopy $W_{t} \subset B(R)$ for $0 \leq t \leq 1$ of the domain $W$ with $W_{0}=W$ and $W_{1} \subset B(S)$. Then we can form a family of manifolds $X_{t}=\mathbb{C} P^{2}(R) \backslash W_{t}$ as before, equip them with almost-complex structures $J_{t}$ varying smoothly with $t$, and study finite energy curves. To fix an asymptotic limit in each $W_{t}$ we let $\eta_{t} \subset \partial W_{t}$ denote the image of $\eta$ under the isotopy. The analysis is exactly the same as for $X$, in particular Lemmas 2.5 and 2.7 hold unchanged. The area formula of Lemma 2.3 remains the same since the isotopy is symplectic. Therefore we can define moduli spaces $\mathcal{M}_{t}$ for each $t$.

Next we have the following.

Lemma 2.11 For a suitably chosen $J_{1}$, the moduli space $\mathcal{M}_{1}$ is empty.

The proof of this will rely on a monotonicity theorem.

Lemma 2.12 Let $u: \Sigma \rightarrow \mathbb{C} P^{2}(R) \backslash B(S)$ be a proper holomorphic curve where $\Sigma$ is a Riemann surface and $\mathbb{C} P^{2}(R) \backslash B(S)$ is equipped with its integrable complex structure. Then $\int_{\Sigma} u^{*} \omega \geq R-S$.

Proof of Lemma 2.12 First we observe by the maximum principle that we have $u(\Sigma) \cap \mathbb{C} P^{1}(\infty) \neq \varnothing$. There exist disjoint balls in $\mathbb{C} P^{2}(R)$ with its integrable complex structure embedded both holomorphically and symplectically of capacities $S$ and $R-S$ respectively. Using a holomorphic isometry we may assume that the ball of capacity $S$ is the standard one and that of capacity $R-S$ is centered at a point of $u(\Sigma) \cap \mathbb{C} P^{1}(\infty)$. Then $u(\Sigma)$ passes through the center of a ball of capacity $R-S$ and thus has area at least $R-S$ from the monotonicity theorem for holomorphic curves; see [7, Section 2.3.E'2].

Proof of Lemma 2.11 First note that by Lemma 2.3 curves in $\mathcal{M}$ have area $R-(a+b)$, at least up to an error of order $\delta$. Next, as $W_{1} \subset B(S)$ we can restrict elements of $\mathcal{M}_{1}$ to $\mathbb{C} P^{2}(R) \backslash B(S)$ and as holomorphic curves have positive area any such restriction has area bounded above by $R-(a+b)+\delta$, which, by choosing $\delta$ sufficiently small, 
we may assume is (strictly) bounded above by $R-S$ (as $S<a+b$ ). As $W_{1}$ is disjoint from $\mathbb{C} P^{2}(R) \backslash B(S)$ we may assume that $J_{1}$ restricts to the standard integrable structure here. Later we will require $J_{1}$ to have some regularity properties, these are compatible with choosing $J_{1}$ to be integrable on $\mathbb{C} P^{2}(R) \backslash B(S)$ since only closed curves lie entirely in this region and for these we have automatic regularity anyway. Then we have a contradiction to Lemma 2.12 .

Henceforth, in order to simplify the notation, we will identify our $X_{t}$ with $X$ and think of the $J_{t}$ as a family of almost-complex structures on $X$. Similarly we will just write $\eta$ for the image of $\eta_{t}$ under this identification. Now, with this in mind, we define the universal moduli space

$$
\mathcal{M}=\left\{(u, t) \mid u: \mathbb{C} \rightarrow X, \operatorname{degree}(u)=1, \bar{\partial}_{J_{t}} u=0, u \sim \eta, t \in[0,1]\right\} / G .
$$

By automatic regularity $\mathcal{M}$ is a 1 -dimensional manifold with boundary $\mathcal{M}_{0} \amalg \mathcal{M}_{1}$. Furthermore the natural projection $\mathcal{M} \rightarrow[0,1],(u, t) \mapsto t$, is an immersion since tangent vectors to $\mathcal{M}$ which map to zero, say at $t_{0}$, are exactly elements of the kernel of the linearization of $\bar{\partial}_{J_{t_{0}}}$, which by automatic regularity is necessarily zero dimensional. Thus the projection is a covering map and so if $\mathcal{M}$ is compact then $\mathcal{M}_{0}$ and $\mathcal{M}_{1}$ have the same number of points. Hence Lemmas 2.10 and 2.11 together imply that the moduli space $\mathcal{M}$ is not compact.

In the next section we investigate this using the compactness theorem of [3].

2.3.3 Compactness To analyze the compactness properties of $\mathcal{M}$ we will assume that the family of almost-complex structures $\left\{J_{t}\right\}$ is chosen to be generic. The precise definition is as follows.

Definition 2.13 We will say that a family $\left\{J_{t}\right\}$ of cylindrical almost-complex structures on the $X_{t}$ is generic if every somewhere injective finite energy curve is regular in a corresponding universal moduli space with its asymptotic limits allowed to vary in their Morse-Bott families (after identifying with a Morse-Bott family in $W$ using the isotopy).

Since there are only countably many such moduli spaces, and Definition 2.13 refers only to somewhere injective curves, the collection of generic families $\left\{J_{t}\right\}$ is of the second category in all smooth families. We can also assume that $J_{1}$ is chosen as required for Lemma 2.11; see the note in the proof.

According to [3], any sequence $\left(u_{t}, t\right) \in \mathcal{M}$ has a subsequence converging in the sense of holomorphic buildings. After taking the subsequence our $t$ will converge to some $t_{0}$ 
and the holomorphic building will then be $J_{t_{0}}$-holomorphic. Roughly speaking, a holomorphic building is a union of finite energy curves whose domains are components of a nodal Riemann surface minus the nodes. Matching nodes in the nodal Riemann surface correspond to matching asymptotic limits.

More precisely, in our situation the nodal Riemann surface is a degeneration of the complex plane given by contracting circles. The components of the limiting holomorphic building map to either $X$ or the symplectization $\partial W \times \mathbb{R}$ of $\partial W$. For the components in $\partial W \times \mathbb{R}$, we can distinguish positive and negative punctures in the obvious way. Each positive puncture is matched either to a negative puncture of another component, or to a puncture of a component in $X$. There will be one unmatched asymptotic limit, corresponding to the original puncture in $\mathbb{C}$ which is asymptotic to $\eta$.

It follows from this that the curves in the symplectization layer can be projected to $\partial W$ and glued along their matching asymptotic limits to produce a map from a (possibly disconnected) Riemann surface whose remaining positive limits are the asymptotic limits of components in $X$ and which has a single remaining negative limit asymptotic to $\eta$.

The following proposition summarizes some immediate properties of a limiting holomorphic building.

Proposition 2.14 The limiting holomorphic building has a single component in $X$. The component is of degree 1 , embedded, has area at most $R-(a+b)$ and virtual dimension at least -1 (in the moduli space of holomorphic curves with a fixed almostcomplex structure but with asymptotic limits allowed to vary in a Morse-Bott family).

Proof The sum of the degrees of the components in $X$ is 1 , but as by Remark 2.4 there are no (nontrivial) components of nonpositive degree, we conclude that the limit has a single component in $X$ of degree 1.

Curves of degree 1 are necessarily somewhere injective. Indeed, if a finite energy curve $u$ is not somewhere injective then it can be written as a composition $u=v \circ p$ where $v$ is another finite energy curve and $p$ is a holomorphic map between the domains, mapping punctures to punctures and having a degree $r>1$. This implies (see the definition of degrees of finite energy curves at the start of Section 2.2.2) that degree $(u)=r$ degree $(v) \neq 1$. Given this, as our curve is a limit of embedded curves in $\mathcal{M}$ it must in fact be embedded.

Given that our curve is somewhere injective, our choice of generic $\left\{J_{t}\right\}$ implies by Definition 2.13 that the curve is regular and so of nonnegative virtual index in its 
universal moduli space. This means that the virtual index in the moduli space with a fixed almost-complex structure is at least -1 .

Finally, as area is preserved in the limit and curves of $\mathcal{M}$ have area $R-(a+b)$, the component in $X$ has area at most $R-(a+b)$.

The area estimate of Proposition 2.14 implies that the component in $X$ is of one of those types listed in Lemma 2.5. The index bound implies by Lemma 2.7 that the curve must be of type (II), (III), (IV) or (V). We deal with these separately.

If the limit is of type (V) then it is asymptotic to an orbit $\gamma_{1,1}$ and any components in $\partial W \times \mathbb{R}$ have area 0 . Here area is measured with respect to the pull back to $\partial W \times \mathbb{R}$ of the restriction of our symplectic form to $\partial W$; it is 0 since all $\gamma_{1,1}$ orbits bound disks of exactly the same area. Thus these components must be trivial cylinders (these are the only curves with area 0$)$, the $\gamma_{1,1}$ orbit is actually $\eta$ and $\left(u_{t}, t\right)$ converge to an element of $\mathcal{M}$.

Suppose now that this component is of type (IV). Then the components in $\partial W \times \mathbb{R}$ have a total of two positive ends, asymptotic to $\gamma_{1}$ and $\gamma_{2}$ respectively, and a negative end asymptotic to $\eta$. As all such components have nonnegative area and both $\gamma_{1}$ and $\gamma_{2}$ have smaller action than $\eta$ we conclude that there must be a single component in $\partial W \times \mathbb{R}$ with two positive ends and a single negative end. But this is impossible as gluing the positive ends of curves in $\partial W \times \mathbb{R}$ to the negative ends of curves in $X$ we must produce a surface of genus 0 , the genus of the $u_{t}$. Gluing the limits as described here results in a genus 1 curve.

Next suppose this component is of type (III). Then the components in $\partial W \times \mathbb{R}$ again have two positive ends, and we can exclude such curves as for type (IV).

Hence if there exists an isotopy with $W_{1} \subset B(S)$ we are left with the conclusion that a sequence $\left(u_{t}, t\right) \in \mathcal{M}$ converges to a holomorphic building whose component in $X$ is of type (II). In other words, for some $t_{0} \in[0,1]$ we have produced a $J_{t_{0}}$-holomorphic finite energy plane asymptotic to $\gamma_{2}^{2}$.

Remark 2.15 Remark 2.6 observed that this is already a contradiction in the case that $b>2 a$, and given Corollary 1.4 this is precisely the case in which we are especially interested.

Despite the above remark, we proceed to prove Theorem 1.1 in its entirety by showing that the bubbling of type (II) curves also leads to a contradiction. We repeat the argument above using type (II) curves instead of type (V) and for $t$ now in the interval $\left[t_{0}, 1\right]$. To be precise, we define moduli spaces

$$
\mathcal{N}_{t}=\left\{u: \mathbb{C} \rightarrow X \mid \operatorname{degree}(u)=1, \bar{\partial}_{J_{t}} u=0, u \sim \gamma_{2}^{2}\right\} / G
$$


and a universal moduli space

$$
\mathcal{N}=\left\{(u, t) \mid u: \mathbb{C} \rightarrow X, \text { degree }(u)=1, \bar{\partial}_{J_{t}} u=0, u \sim \gamma_{2}^{2}, t \in\left[t_{0}, 1\right]\right\} / G .
$$

Again we see that by hypothesis $\mathcal{N}_{t_{0}}$ is nonempty, and $\mathcal{N}_{1}$ is empty as in Lemma 2.11. Also, as with $\mathcal{M}$, the adjunction formula can be used to show that curves in $\mathcal{N}$ are embedded. (More generally, in dimension 4 any limit of embedded curves which is not a multiple cover is also embedded.) Thus Theorem 2.9 can also be applied here to give automatic regularity for curves in $\mathcal{N}$. Hence the projection $\mathcal{N} \rightarrow[0,1],(u, t) \mapsto t$ is a covering map.

However now the 1-dimensional manifold $\mathcal{N}$ is compact, and this gives a contradiction. Indeed, if there is a loss of compactness we must see curves of types (I), (III), (IV) or (V) in $X$. But type (I) can be still be excluded by regularity, and type (III) in exactly the same way as above. Finally curves of types (IV) and (V) have strictly larger area than curves of type (II) as $b>a$ and so do not appear either.

Remark 2.16 As mentioned in the introduction, and described explicitly in Section 3, if $R>2 a+b$ and $2 a<b$ then there are Hamiltonian diffeomorphisms with compact support in $B(R)$ mapping $P(a, b)$ into a ball $B(S)$ with $S<a+b$. It is interesting to observe the behavior of our finite energy curves in this situation. The moduli spaces $\mathcal{M}_{0}, \mathcal{M}_{1}$ and the universal moduli space $\mathcal{M}$ can be defined as before and curves in these spaces have area $R-(a+b)$. As before $\mathcal{M}_{0}$ is nonempty while $\mathcal{M}_{1}$ is empty and $\partial \mathcal{M}=\mathcal{M}_{0} \amalg \mathcal{M}_{1}$. But as $R>2 a+b$ we cannot exclude planes of degree 1 asymptotic to a hyperbolic orbit $\gamma_{2,1}$. By Lemma 2.3 these have area roughly $R-(2 a+b)$. The virtual index is -1 (see the proof of Lemma 2.7) and so typically such curves can appear in generic 1-parameter families of almost-complex structures. It is degeneration onto these curves which causes the loss of compactness of $\mathcal{M}$ and prevents our arriving at a contradiction.

\section{Symplectic folding}

Here we establish Theorem 1.3 by following the symplectic folding construction while minimizing the size of the balls in which the support of our various diffeomorphisms lie. It clearly suffices to find a Hamiltonian diffeomorphism with support in an arbitrarily small neighborhood of $B(2 a+b)$ mapping $P(a, b)$ arbitrarily close to $B(2 a+b / 2)$.

For convenience we will use symplectic polar coordinates $(R, \theta)$ on the $\left(x_{2}, y_{2}\right)$ plane, where $R=\pi\left(x_{2}^{2}+y_{2}^{2}\right)$ and $\tan \theta=\frac{y_{2}}{x_{2}}$. Therefore we can write a disk of area $a$ in this plane as $D(a)=\{R \leq a\}$. 


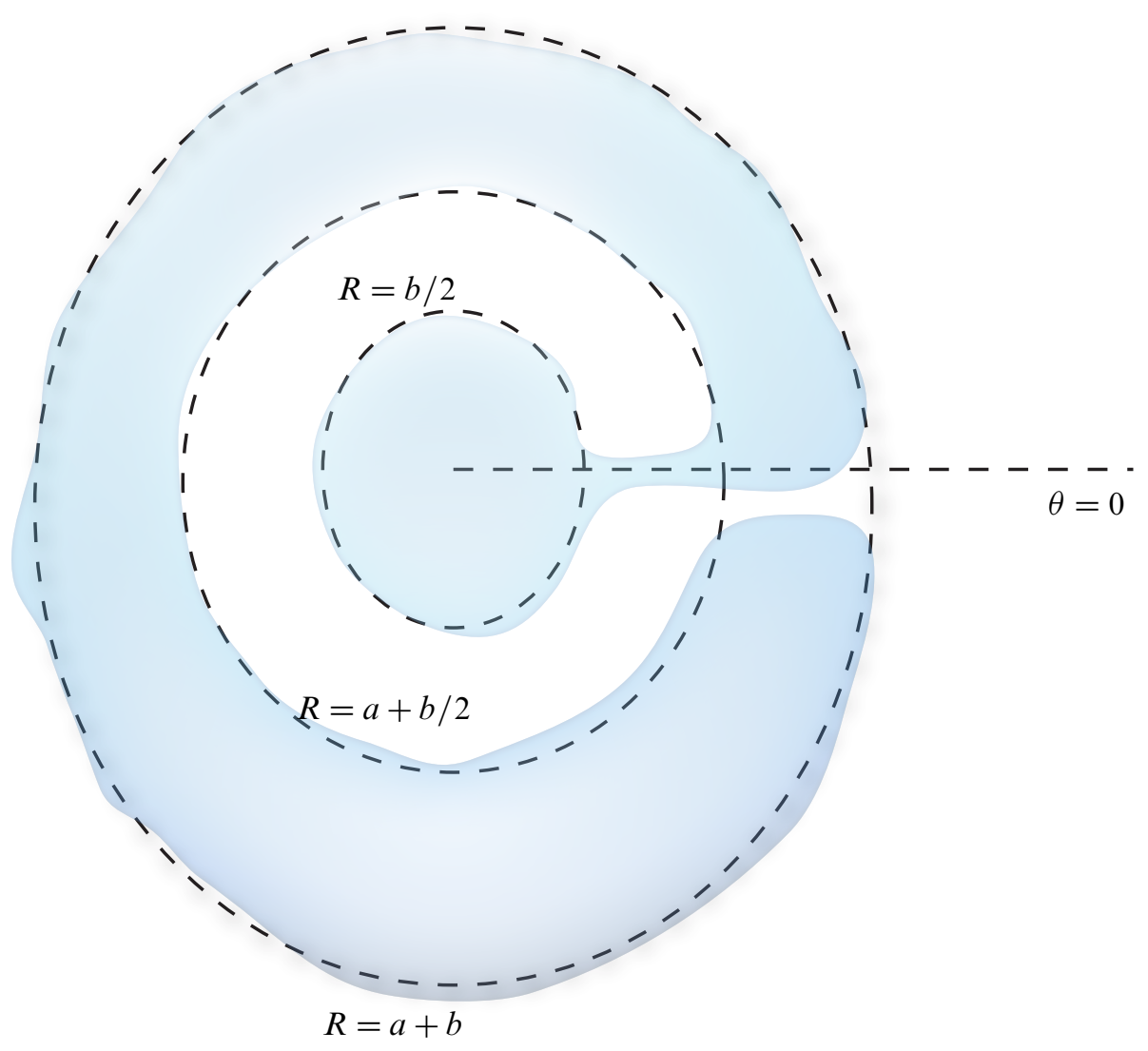

Figure 1: The image of $D(b)$ in the $(R, \theta)$ plane

We start with a polydisk $P(a, b)=D(a) \times D(b)$ where $D(a)$ and $D(b)$ denote disks in the $\left(x_{1}, y_{1}\right)$ and $\left(x_{2}, y_{2}\right)$ planes respectively. This polydisk lies inside $B(a+b)$.

Step 1: Repositioning $D(b)$ We apply a symplectomorphism $\psi_{1}$ of the $(R, \theta)$ plane mapping $D(b)$ to an arbitrarily small neighborhood of

$$
U=\left\{R \leq \frac{b}{2}\right\} \cup\left\{\theta=0, \frac{b}{2} \leq R \leq a+\frac{b}{2}+\delta\right\} \cup\left\{a+\frac{b}{2}+\delta \leq R \leq a+b+\delta\right\} .
$$

We can take $\delta$ arbitrarily small, but for the following steps to apply it must be strictly positive. Figure 1 is a sketch of the image of $D(b)$.

Now for Step 1 we apply the symplectomorphism $\phi_{1}=\mathrm{id} \times \psi_{1}$ to the polydisk $P(a, b)$. As $U$ lies in a disk $D(a+b+\delta)$ the image of $P(a, b)$ lies in a ball $B(a+b+\delta+a)$ and we can realize $\phi_{1}$ as a Hamiltonian diffeomorphism with support arbitrarily close to $B(2 a+b)$. Let $P_{1}=\phi_{1}(P(a, b))$. 


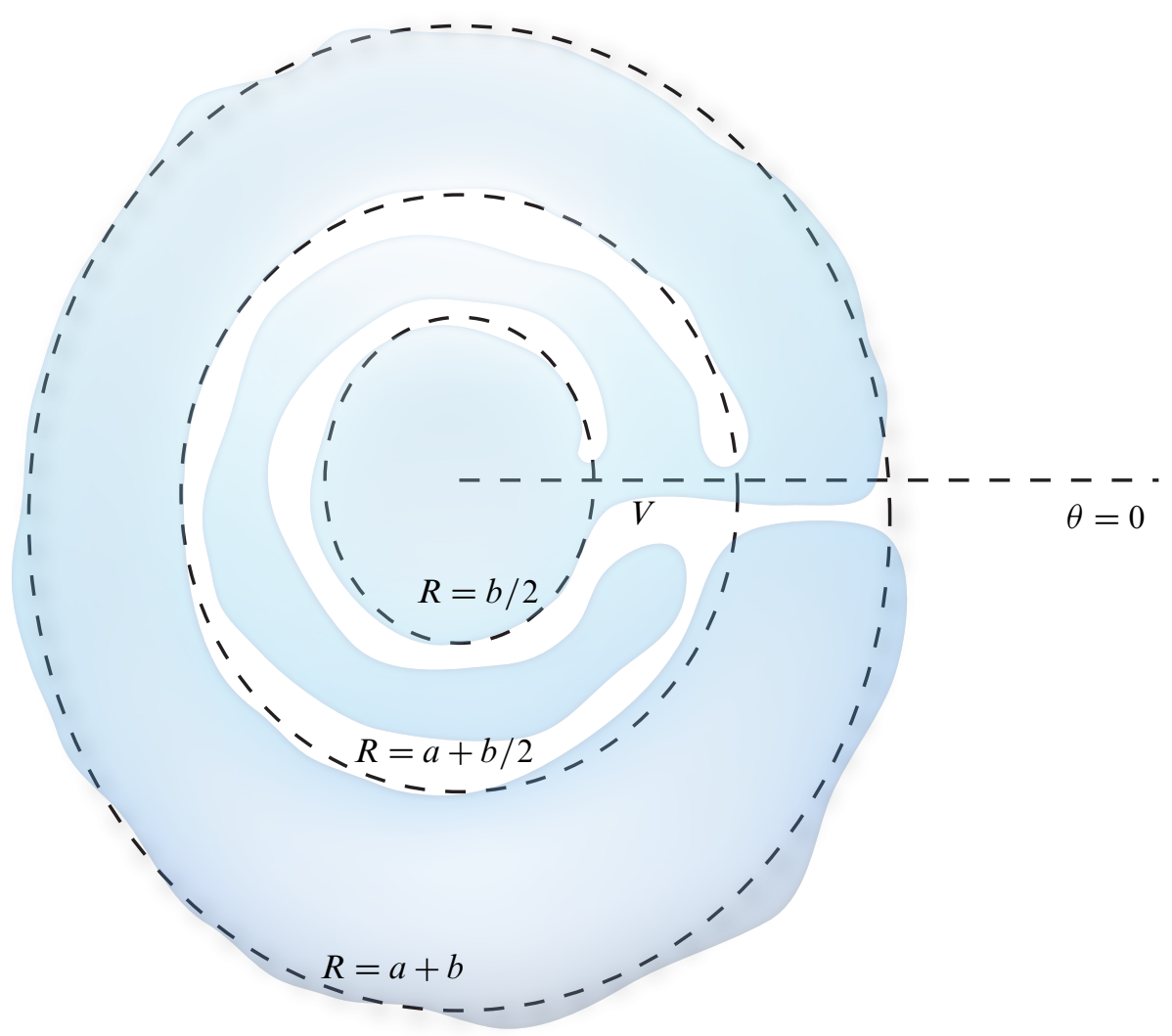

Figure 2: The projection of $P_{2}$ to the $(R, \theta)$ plane

Step 2: Displacing $\boldsymbol{D}(\boldsymbol{a}) \times \boldsymbol{D}(\boldsymbol{b} / \mathbf{2})$ outward There exists a Hamiltonian diffeomorphism $\psi_{2}$ displacing $D(a)$ from itself and having compact support in a small neighborhood of $D(2 a)$. Let $H_{2}\left(x_{1}, y_{1}\right)$ be a generating Hamiltonian, that is, $\psi_{2}$ is the time-1 flow of the Hamiltonian vector field corresponding to $\mathrm{H}_{2}$. We may assume that $0 \leq H_{2} \leq a$ and it is also not hard to arrange that for all $0<\lambda<1$ the Hamiltonian diffeomorphism generated by $\lambda H_{2}$ maps $D(a)$ into a neighborhood of $D((1+\lambda) a)$.

Define $\chi(R)$ to be a decreasing function equal to 1 when $R \leq b / 2$ and 0 when $R \geq a+b / 2+\delta$ and having slope bounded by $\frac{1}{a}$.

Our second step is to apply the Hamiltonian diffeomorphism $\phi_{2}$ which is generated by $\chi(R) H_{2}\left(x_{1}, y_{1}\right)$. Set $P_{2}=\phi_{2}\left(P_{1}\right)$. We examine separately the images of points with $\{R \leq b / 2\},\{b / 2 \leq R \leq a+b / 2+\delta\}$ and $\{a+b / 2+\delta \leq R \leq a+b+\delta\}$ under this flow. As the generating Hamiltonian is independent of $\theta$ the flow preserves the $R$ 
coordinate and in particular these regions. Figure 2 is a sketch of the projection of $P_{2}$ to the $(R, \theta)$ plane; the figure is justified by the following analysis.

- If $R \leq b / 2$ then the flow fixes $(R, \theta)$ and the $\left(x_{1}, y_{1}\right)$ coordinates remain in a neighborhood of a disk $D(2 a)$. Thus the flow remains close to a ball $B(2 a+b / 2)$.

- If $b / 2 \leq R \leq a+b / 2+\delta$ then, as the flow of $\lambda H_{2}\left(x_{1}, y_{1}\right)$ maps $D(a)$ into a neighborhood of $D((1+\lambda) a)$, the trace of the $\left(x_{1}, y_{1}\right)$ coordinates throughout lie in a neighborhood of a disk $D((1+\chi(R)) a)$. As $\chi$ is roughly $(a+b / 2-R) / a$, this disk is roughly equal to $D(2 a+b / 2-R)$. Therefore, as the corresponding $\left(x_{2}, y_{2}\right)$ coordinates lie in $\partial D(R)$, the flow remains close to the ball $B(2 a+b / 2)$. Meanwhile the rate of increase of the $\theta$ coordinate is bounded by $2 \pi \chi^{\prime}(R) H_{2}\left(x_{1}, y_{1}\right)<2 \pi$. Hence we may assume that the projection of the image to the $(R, \theta)$ plane continues to avoid a narrow segment $V$ just below the $\{\theta=0\}$ axis as shown in Figure 2 .

- Finally, the flow is constant in the region $\{a+b / 2+\delta \leq R \leq a+b\}$.

Step 3: Tucking the outer ring $P_{\mathbf{2}} \cap\{R \geq a+b / 2\}$ under the center Here we apply a symplectomorphism $\phi_{3}$ generated by a Hamiltonian $H_{3}(R, \theta)$ with support in a neighborhood of $D(a+b+\delta)$ only to points of $P_{2}$ which lie in the region $\{a+b / 2+\delta \leq R \leq a+b+\delta\}$. The flow of $H_{3}$ will fix points close to the boundary $\{a+b / 2+\delta=R\}$ of this region and so can be extended as a constant to the rest of $P_{2}$. In fact, we will take $H_{3}$ to be identically 0 on the region $\{b / 2 \leq R \leq a+b / 2+\delta\} \backslash V$.

We choose $\mathrm{H}_{3}$ such that the corresponding flow moves all of $P_{2} \cap\{a+b / 2+\delta \leq$ $R \leq a+b+\delta\}$ to a neighborhood of $\{R \leq b / 2\} \cup V$. Such an $H_{3}$ exists because $\{a+b / 2+\delta \leq R \leq a+b+\delta\}$ has area roughly $b / 2$ and we may assume that the projection of $P_{2} \cap\{a+b / 2+\delta \leq R \leq a+b+\delta\}$ to the $(R, \theta)$ plane is simply connected; see Figure 1 . Then $H_{3}$, thought of as a Hamiltonian on $\mathbb{R}^{4}$, can be used to define a symplectomorphism $\phi_{3}$ of $P_{2}$ once we check that the traces of points moved by the flow of $H_{3}$ are disjoint from the points of $P_{2}$ where we are defining $\phi_{3}$ to be the identity. This follows since the only possible intersections lie in $\{R \leq b / 2\}$, but the $\left(x_{1}, y_{1}\right)$ coordinates of points in $P_{2} \cap\{R \leq b / 2\}$ lie outside $D(a)$ while those of points in $P_{2} \cap\{a+b / 2+\delta \leq R \leq a+b+\delta\}$ lie in $D(a)$ (and $\phi_{3}$ fixes our $\left(x_{1}, y_{1}\right)$ coordinates). Hence we have a well-defined $\phi_{3}$, a symplectic folding map. The only points moved by the flow have $\left(x_{1}, y_{1}\right) \in D(a)$ and $R \leq a+b+\delta$, and so the Hamiltonian isotopy remains in an arbitrarily small neighborhood of $B(2 a+b)$.

Finally, we recall that points of $P_{2} \cap\{R \leq a+b / 2+\delta\}$ all lie in a neighborhood of $B(2 a+b / 2)$, and since they fixed by $\phi_{3}$ the image $P_{3}=\phi_{3}\left(P_{2}\right)$ lies in the union of a neighborhood of $B(2 a+b / 2)$ together with $\phi_{3}\left(P_{2} \cap\{a+b / 2+\delta \leq R \leq a+b+\delta\}\right)$. This second part of the image lies in a neighborhood of $D(a) \times\{R \leq a+b / 2+\delta\}$ which in turn lies also in a neighborhood of $B(2 a+b / 2)$. 
In summary, our isotopy has support in a neighborhood of $B(2 a+b)$ (we already approach the boundary of this region in Step 1), and the resulting diffeomorphism has image close to $B(2 a+b / 2)$ as required.

\section{References}

[1] F Bourgeois, A Morse-Bott approach to contact homology, $\mathrm{PhD}$ thesis, Stanford University (2002) MR2703292 Available at http://search.proquest.com/docview/ 305591502

[2] F Bourgeois, A Morse-Bott approach to contact homology, from: "Symplectic and contact topology: Interactions and perspectives", (Y Eliashberg, B Khesin, F Lalonde, editors), Fields Inst. Commun. 35, Amer. Math. Soc. (2003) 55-77 MR1969267

[3] F Bourgeois, Y Eliashberg, H Hofer, K Wysocki, E Zehnder, Compactness results in symplectic field theory, Geom. Topol. 7 (2003) 799-888 MR2026549

[4] I Ekeland, H Hofer, Symplectic topology and Hamiltonian dynamics, II, Math. Z. 203 (1990) 553-567 MR1044064

[5] Y Eliashberg, A Givental, H Hofer, Introduction to symplectic field theory, Geom. Funct. Anal. (2000) 560-673 MR1826267

[6] A Floer, H Hofer, K Wysocki, Applications of symplectic homology, I, Math. Z. 217 (1994) 577-606 MR1306027

[7] M Gromov, Pseudo-holomorphic curves in symplectic manifolds, Invent. Math. 82 (1985) 307-347 MR809718

[8] H Hofer, V Lizan, J-C Sikorav, On genericity for holomorphic curves in fourdimensional almost-complex manifolds, J. Geom. Anal. 7 (1997) 149-159 MR1630789

[9] H Hofer, K Wysocki, E Zehnder, A characterisation of the tight three-sphere, Duke Math. J. 81 (1995) 159-226 MR1381975

[10] H Hofer, K Wysocki, E Zehnder, Properties of pseudo-holomorphic curves in symplectisations, II: Embedding controls and algebraic invariants, Geom. Funct. Anal. 5 (1995) 270-328 MR1334869

[11] H Hofer, K Wysocki, E Zehnder, Properties of pseudoholomorphic curves in symplectisations, I: Asymptotics, Ann. Inst. H. Poincaré Anal. Non Linéaire 13 (1996) 337-379 MR1395676

[12] H Hofer, K Wysocki, E Zehnder, Properties of pseudoholomorphic curves in symplectizations, III: Fredholm theory, from: "Topics in nonlinear analysis", (J Escher, G Simonett, editors), Progr. Nonlinear Differential Equations Appl. 35, Birkhäuser, Basel (1999) 381-475 MR1725579

[13] M Hutchings, M Sullivan, Rounding corners of polygons and the embedded contact homology of $T^{3}$, Geom. Topol. 10 (2006) 169-266 MR2207793 
[14] F Lalonde, D McDuff, The geometry of symplectic energy, Ann. of Math. 141 (1995) 349-371 MR1324138

[15] D McDuff, Symplectic embeddings of 4-dimensional ellipsoids, J. Topol. 2 (2009) 1-22 MR2499436

[16] D McDuff, D Salamon, J-holomorphic curves and symplectic topology, American Mathematical Society Colloquium Publications 52, Amer. Math. Soc. (2004) MR2045629

[17] J Robbin, D Salamon, The Maslov index for paths, Topology 32 (1993) 827-844 MR1241874

[18] F Schlenk, Embedding problems in symplectic geometry, de Gruyter Expositions in Mathematics 40, Walter de Gruyter, Berlin (2005) MR2147307

[19] M Schwarz, Cohomology operations from $S^{1}$-cobordisms in Floer homology, $\mathrm{PhD}$ thesis, ETH Zürich (1995)

[20] C Wendl, Automatic transversality and orbifolds of punctured holomorphic curves in dimension four, Comment. Math. Helv. 85 (2010) 347-407 MR2595183

Department of Mathematics, University of Notre Dame 255 Hurley, Notre Dame, IN 46556, USA

hind.1@nd.edu

http://math.nd.edu/people/faculty/richard-k-hind/

Received: 31 October 2012 Revised: 4 February 2013 